\title{
Mathematical and computational tools in chemistry: CMMSE-2014
}

\author{
J. Vigo-Aguiar · Higinio Ramos
}

Published online: 22 January 2015

(C) Springer International Publishing Switzerland 2015

It is our pleasure to present to the readers this Special Issue of the Journal of Mathematical Chemistry. It contains selected papers from those presented at the 14th International Conference of Computational and Mathematical Methods in Science and Engineering, CMMSE_-2014, held at Barceló Costa Ballena Hotel, Rota, Cádiz, Spain, between 3 and 7 of July 2014.

This Special Issue continues a well established and fruitful tradition of producing special issues of the Journal of Mathematical Chemistry for the CMMSE conference series that dates back to 2002.

There exists a close relationship between the need of solving real problems in various fields of science and the mathematical formulation and approximation of the problems posed. Usually, physics and engineering have been the more traditional fields of applications of mathematics, however, for some years now, chemical sciences have also reached a similar stage in the application of mathematical procedures for their development. The large number of papers presented at our conferences, addressing topics related to chemistry, provide a sample, all indicating the increasing research activity in the field.

One significant paper is by J.M. García-Martínez et al., who apply the methodology of the Universal Evolutionary Global Optimization approach, UEGO, to solve the protein structure optimization problem based on the HP model. The authors have designed a new and efficient local optimizer based on the characteristics of the protein model.

\footnotetext{
J. Vigo-Aguiar $(\varangle)$

Universidad de Salamanca, Salamanca, Spain e-mail: jvigo@usal.es

H. Ramos

Escuela Politécnica Superior, Zamora, Spain

e-mail: higra@usal.es
} 
The article "Behaviour of fixed and critical points of the $(\alpha, c)$-family of iterative methods" by B. Campos et al., analyzes the dynamical behavior of third-order iterative root-finding methods for a family of problems dependent on two parameters. The importance of this study is related to the search for the most stable elements of the family. Iterative methods are commonly used for solving some nonlinear equations that appear in chemistry, such as those in the reaction-diffusion equations that arise in autocatalytic chemical reactions.

The article by C. Calvo-Jurado and W. J. Parnell presents a straightforward mechanism for the construction of the Hashin-Shtrikman bounds for transversely isotropic composites, focusing on the two-phase limiting cases cosidered in the conductivity setting. Of specific interest in composite design is the fact that the shape of the inclusions and their distribution can be specified independently.

The research conducted by Rajnesh Lal and Zhenquan Li investigates the sensitivity of a mesh refinement method against the accuracy of numerical solutions of the 2-D steady incompressible lid-driven flow relative to a collocated finite volume method. The method considered can be applied to incompressible fluids or steady state fluid flows or mass and heat transfers.

The paper "The variational spline method for solving Troesch's problem", by A. Kouibia et al., considers a variational approximation method for solving the wellknown Troesch's problem. The authors construct a sequence of approximate solutions and prove that it converges to the exact solution. This problem appears extensively in engineering and science, including the modeling of reaction-diffusion and heat transfer processes.

The article by J. L. Hueso et al. focuses on the problem of approximating multiple roots of nonlinear equations. Multiple roots appear in various applications, such as the compression of band-limited signals and the multifactor effect in electronic devices. The proposed methods are optimal because they reach fourth order of convergence and only use three function evaluations per step. Adding one functional evaluation, they also obtain a family of derivative-free iterative methods.

In the article, "A new fourth-order family for solving nonlinear problems and its dynamics", a new parametric class of Jarratt-type iterative methods for solving nonlinear systems of equations is proposed by Alicia Cordero, Licheng Feng, Alberto Magreñán and Juan R. Torregrosa. A dynamical analysis of low-degree polynomials is carried out in order to choose those elements of the family which have better conditions of stability. The results are checked by solving the nonlinear system that arises from the partial differential equation of certain molecular interactions.

B. Pekmen and M. Tezer-Sezgin present a study of the dual reciprocity boundary element (DRBEM) solution of the Brinkman-Forchheimer-extended Darcy model in a porous medium containing an incompressible, viscous fluid. The governing dimensionless equations are solved in terms of stream function, vorticity, and temperature. The numerical study shows that the effects of Darcy number, Rayleigh number, and the porousity of the porous medium on natural convection are in accordance with the natural convection flow behaviors investigated.

The article "Sensitivity Analysis of a Linear and Unbranched Chemical Process with n Steps", by L. Bayón et al., supposes a generalization of the optimal control problem that arises when considering a linear, unbranched chemical process with $n$ 
steps. A quasi-analytical solution is provided considering the minimization of the operation time and the non-equal catalytic efficiencies of the enzymes.

The research underlying the article by $\mathrm{M}$. Rosa et al., "A conservation law for a generalized chemical Fisher equation", proposes to study a generalized Fisher equation with variable coefficients from the point of view of the theory of symmetry reductions in partial differential equations. There is a considerable interest in finding conservation laws for nonlinear evolution equations which have no Lagrangians, and the authors have constructed some nontrivial conservation laws for a generalized Fisher equation.

The use of microwave radiation for heating is common in many industrial situations such as cooking, sterilizing, melting, smelting, sintering, and drying. Microwaves are widely used in chemical industry to accelerate chemical reactions. In the article by R. de la Rosa et al. the authors have determined the subclasses of a family of equations describing microwave heating, which are self-adjoint and nonlinearly self-adjoint, and the authors have derived conservation laws for some of these partial differential equations.

Finally, the paper "Numerical modelling transient current in the time-of-flight experiment with time-fractional advection-diffusion equations", by L. F. Morgado and M. L. Morgado, develops an implicit numerical method for the approximate solution of a general time-fractional advection-dispersion equation. This equation arises in many physical models where anomalous dispersion occurs with an additional velocity field and under the influence of an external force. An example of this phenomena is the transient electrical current of the time of flight experiment of disordered semiconductors.

The Editors are grateful to all the authors and referees for their valuable contributions to this special volume. Also, a special mention is due to Professor Paul G. Mezey, Editor-in-Chief, for his contibutions. 